\title{
Mucositis in irradiated cancer patients: Effects of an antiseptic mouthrinse
}

\author{
Isabel Lanzós ${ }^{1}$, David Herrera ${ }^{2}$, Sagrario Santos ${ }^{3}$, Ana O'Connor ${ }^{4}$, Carmen Peña ${ }^{5}$, Eduardo Lanzós ${ }^{6}$, Ma- \\ riano $\operatorname{Sanz}^{7}$
}

\footnotetext{
${ }^{1}$ Master in Periodontology, Faculty of Odontology, University Complutense, Madrid, Spain

${ }^{2}$ Full-time Professor, ETEP Research Group, University Complutense, Madrid, Spain

${ }^{3}$ Master in Periodontology, Faculty of Odontology, University Complutense, Madrid, Spain

${ }^{4}$ Laboratory Technician, Faculty of Odontology, University Complutense, Madrid, Spain

${ }^{5}$ Adjunct Physician, Oncological Radiotherapy Service, Hospital 12 de Octubre, Madrid, Spain

${ }^{6}$ Head of Service, Oncological Radiotherapy Service, Hospital 12 de Octubre, Madrid, Spain

${ }^{7}$ Full-time Professor, ETEP Research Group, Complutense University, Madrid, Spain
}

\author{
Correspondence: \\ Faculty of Odontology \\ Universidad Complutense \\ Plaza Ramón y Cajal, s/n \\ 28040 Madrid, Spain \\ davidher@odon.ucm.es
}

Received: $24 / 10 / 2009$

Accepted: 24/12/2009

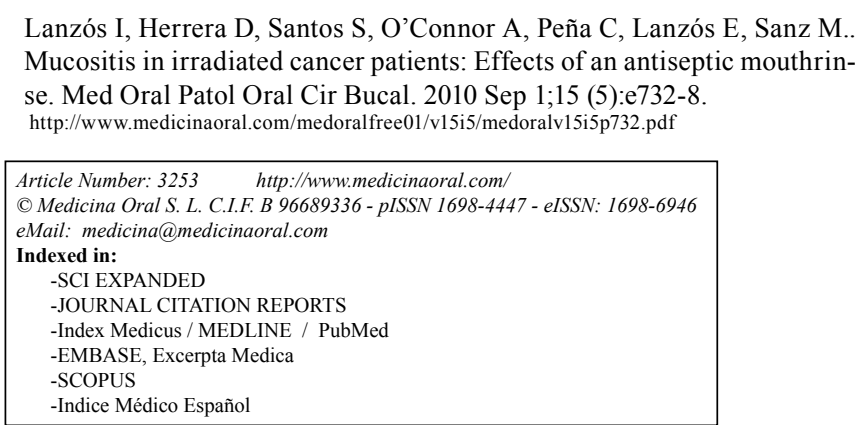

\begin{abstract}
Objective: To assess the effects of an antiseptic, non-alcohol based mouth-rinse containing chlorhexidine and cetylpyridinium chloride, in preventing the oral complications associated to radiation therapy in head-and-neck cancer patients.

Study design: This was a parallel, double blind, prospective, randomized clinical trial. Cancer patients were randomly assigned to one of the two treatments (test mouth-rinse or a placebo). Three visits were scheduled (baseline, 14 and 28 days). Different outcome variables were evaluated: mucositis, plaque and gingival indices, stimulated saliva and salivary $\mathrm{pH}$.

Results: 70 patients were screened and 36 were included. The presence and the degree of mucositis significantly increased in both groups and no significant differences were detected between groups, although the median increase in the placebo group (1.81) at 2 weeks was higher than in the test group (1.20).

Conclusions: Within the limitations of the small sample size, this study suggests that the use of the tested mouthrinse may lead to some improvements in clinical parameters in patients irradiated for head-and-neck cancer.
\end{abstract}

Key words: Mucositis, chlorhexidine, cetylpyridinium chloride, head-and-neck tumour, radiotherapy. 


\section{Introduction}

Every year, approximately 1.3 millions of Europeans are diagnosed of cancer and more than 0.8 millions die of this disease. In Spain, cancer is the main cause of death ( 90.000 cancer patients per year, $25 \%$ of all deaths), according to the information provided by the Ministry of Health in 2006. Oral cancer represents $2-4 \%$ of all diagnosed cancers in Spain, and its prevalence ranges between $11.4-17.4 \%$ per 100000 inhabitants. Among the intraoral locations, the tongue (25.1\%) and the floor of the mouth (10.2\%) are the most common.

Treatment for oral cancer (either radiotherapy and/or chemotherapy) is becoming increasingly effective, but it is associated with both short and long-term secondary side effects. Among these, the most frequent is oral mucositis (OM) (1).

Radio-induced OM develops in almost all patients treated for cancers in the mouth, oropharynx or nasopharynx, and in two thirds of the cancers in the larynx or hypopharynx $(2,3)$. The risk of OM and its severity is related to the characteristics of the radiation therapy, such as dose, field size and fractionation, as well as to the patient's oral hygiene during therapy. Hyper-fractionation and combination with chemotherapy increase the prevalence, severity and duration of $\operatorname{OM}(2,4)$.

Radio-induced OM affects mainly non-queratinized oral mucosa, such as lip and buccal mucosae, lateral and anterior mucosae of the tongue, floor of the mouth, and, less frequently, hard palate mucosa $(2,4)$. Early signs of OM include erythema that appears after approximately $10 \mathrm{~Gy}$ of cumulative radiation dosage for head and neck $(\mathrm{H} \& \mathrm{~N})(4)$. After 7-10 days, or a cumulative dosage of $30 \mathrm{~Gy}$, ulcers are detected, associated to discomfort and changes in patient dietary habits $(2,4)$. Radio-induced OM lasts for at least 2 weeks after radiation therapy have finished $(2,4)$. The impact of OM can be very important, since patients may need hospitalization or even modifications in their cancer therapy $(1-4,5)$.

Stokman and coworkers (2), in a meta-analysis of 45 studies and 8 interventions, concluded that "to date, no single intervention completely prevents oral mucositis". However, they identified treatments with significant preventive effect, such as PTA (combination of the antimicrobials polymyxin E, tobramycine and amphotericin B), GM-CSF (granolocyte-macrophage colonystimulating factor), oral cooling by means of ice chips, and amifostine. More recently, two systematic reviews on preventive (6) (including 89 studies and 29 interventions) and on therapeutic approaches (7) (with 26 studies) concluded that some benefit could be obtain with certain strategies, such as: amifostine, natural remedies from chinese medicine and hydrolytic enzymes, especially in the prevention and the reduction of its severity. It has been suggested, therefore, that the best approach may be the use of a combined preventive therapy strat- egy. Following this concept, protocols of oral care for patients in radiotherapy for $\mathrm{H} \& \mathrm{~N}$ tumours have developed, including the use of soft tooth brushes, periodic oral health assessments and follow up and the inclusion of dentists in the multidisciplinary team (8).

The mouth of patients under radiotherapy for $\mathrm{H} \& \mathrm{~N}$ cancer should be carefully supervised, even before the initiation of the therapy, with the goal of achieving low plaque and gingivitis levels. During therapy, when mechanical plaque control might be difficult or inadequate, chemical plaque control may be beneficial. Among the agents used in chemical plaque control, chlorhexidine (CHX) is the most active, due to its wide spectrum (including yeasts) and high substantivity. Its safety has been proved in long-term studies, although the occurrence of side effects is frequent, including tooth staining and soft tissue and taste alterations. Studies with CHX have demonstrated its capacity to prevent oral complications, such as the occurrence of chronic or opportunistic infections, including Candida sp. Infections, in high risk patients (irradiated patients, patients in chemotherapy or bone marrow transplant recipients) (for review, see (9)).

Usage of CHX mouth rinses may reduce the bacterial and fungal colonization in the mouth (9), and since gram-negative anaerobic bacterial species may have a role in the pathogenesis of OM (10), CHX thus may help to prevent or decrease the severity of OM. Some studies with different designs have already tested the hypothesis (11-15) with variable results that make not possible to confirm or reject the hypothesis (6).

In the last years, new CHX formulations have been marketed, with the goal of reducing secondary effects (e.g. by excluding alcohol from the formulation), or increasing its antimicrobial effect (e.g. by adding other active agents). Among these new formulations, a mouth rinse that combines CHX with cetyl-pyridinium chloride (CPC), and no alcohol, has been tested (16), demonstrating an increase in antibacterial activity. Due to the lack of alcohol and to the increased antimicrobial activity, we hypothesized that the use of this specific mouth rinse may be beneficial for the oral health status in irradiated patients for $\mathrm{H} \& \mathrm{~N}$ cancer.

Thus, the aim of this randomised clinical trial was to assess the effects of an antiseptic mouth rinse, with $\mathrm{CHX}$ and CPC and no alcohol, in the prevention of oral complications associated to irradiation in $\mathrm{H} \& \mathrm{~N}$ cancer patients.

\section{Patients and Methods}

\section{Patients}

Consecutive patients were selected at the Oncology Radiotherapy Service at the "12 de Octubre" Hospital (Madrid), using the following criteria:

Inclusion criteria: irradiated as part of the therapy of 
head-and-neck cancer; aged 18-75; at least 10 teeth; informed consent.

Exclusion criteria: patients already diagnosed of suffering a mucosal pathology, such as lichen or lupus; pregnant women; patients with orthodontic therapy.

\section{Methods}

Study design

The study was a parallel, double-blind, prospective, randomised clinical trial.

Patients were screened for compliance with the inclusion and exclusion criteria and once they agreed to participate by signing the IRB approved informed consent; they were entered in the study and appointed for the baseline visit.

The study consisted on three visits:

- Visit 1 or baseline: the day when radio-therapy was started.

- Visit 2: 14 days after baseline.

- Visit 3 or final visit: 28 days after baseline.

- Visit 1. Baseline.

Patients were examined, outcome variables were recorded and samples for microbiology were collected. The following outcome variables were recorded: degree of mucositis, plaque and gingival indices, presence of caries and amounts and $\mathrm{pH}$ of saliva.

After this evaluation, all participating patients were randomised and the treatments were allocated by providing the assigned mouth rinse together with the instructions for use. They were then appointed for the next visit according to the study plan.

-Visit 2. 14-day evaluation.

The same sampling and registration of outcome variables were done again, together with an interview with the patient assessing their compliance in using the assigned mouth rinse and the occurrence of any adverse event.

- Visit 3. Final visit after 28 days.

Identical to visit 2, the sampling, registration of outcome variables, compliance and occurrence of any adverse event were carried out.

All the outcome variables were assessment by a single and calibrated examiner, who was blind to the treatment assignment.

\section{Outcome variables}

Evaluation of Mucositis. The Scale of the Radiation Therapy Oncology Group/European Organization Research and Treatment of Cancer (RTOG/EORTC) was utilized. This scale assesses the presence of mucositis in different degrees based on the intensity of the affectation, from no lesion (0) to the occurrence of ulceration and/or necrosis (4).

The following criteria categorize this scale from $0-4$ :

0 . No lesion.

1. Eritema; may experience mild pain not requiring analgesic.
2. Patchy mucositis that may produce an inflammatory sero-sanguinitis discharge; it may experience moderate pain.

3. Confluent fibrinous mucositis; may include severe pain requiring narcotics

4. Ulceration, haemorrhage, or necrosis.

Plaque index

The index proposed by O'Leary and coworkers on 1972 was selected: four surfaces were evaluated per tooth in all teeth. The number of positive surfaces were multiplied for 100, and divided by the total number of surfaces evaluated $(\mathrm{IP}=$ plaque positive surfaces / total surfaces).

Gingival index

The index proposed by Ainamo and Bay in 1975 was selected, and it was assessed in four surfaces per tooth in all teeth, as present or absent.

Amount of stimulated saliva (mL)

Sampling of saliva was done through the "expectoration technique". The patients were provided with paraffin that was chewed during 5 minutes. Then they are asked to spit all produced saliva in a graduated tube and the rate of salivary flow is calculated, in $\mathrm{mL}$ per minute.

Salivary $\mathrm{pH}$

A small amount of the saliva was collected by means of a pipette and a drop was placed in the yellow part of the Dentobuff Strip ${ }^{\circledR}$ (Orion Diagnostica, Finland), and let to dry for $5 \mathrm{~min}$. Then, the change in colour was evaluated and registered and the salivary $\mathrm{pH}$ was calculated. The yellow colour (low buffer capacity, $\mathrm{pH}$ of 4 or lower) was coded as 1 , green colour (moderate buffer capacity, $\mathrm{pH}$ between 4.5 and 5.5) as 2, and the blue colour (high buffer capacity, pH 6 or higher) as 3 .

\section{Treatments}

Patients included in the study were randomly assigned to one of the two treatments, either test or control. Randomisation was done through a computer-generated list that assigned treatments by numbers. Patients received a number after inclusion, corresponding to a numerically coded mouth rinse. The list and the numbered bottles were provided by the promoter, and the assignation of numbers was made by the researchers in consecutive order. Codes were not opened until the end of the study. Both patients and researchers were blinded throughout the study.

Patients in the test group rinsed with Perio-Aid Tratamiento ${ }^{\circledR}$ (Dentaid, Cerdanyola del Valles, Spain) composed of $0.12 \% \mathrm{CHX}$ and $0.05 \% \mathrm{CPC}$ as active ingredients. Patients in the control group rinsed with a placebo mouth rinse, identical to the test product but without the active components. Both formulations lacked any alcohol.

All patients received written instructions on the use of their assigned treatment. In brief, they should carry out their usual tooth-brushing and oral hygiene proce- 
dures, and then they should rinse with $15 \mathrm{~mL}$ of the assigned product, for 30 second, twice a day (morning and evening).

Statistical analyses

The study primary outcome was the occurrence and the changes in the degree of mucositis according to the described scale. These changes were evaluated by means of the chi-square test with the Yates's correction. Comparisons between groups were assessed by means of the Mann-Whitney test.

As secondary outcome variables, plaque and gingival indices were evaluated inter- (both at each visit and in changes between visit) and intra-group. Intergroup changes were compared by means of the Mann-Whitney test, while intra-group changes were studied with the Wilcoxon test.

The changes in the amount of saliva in $\mathrm{mL}$ were assessed by the t-test. The salivary $\mathrm{pH}$ was evaluated in an identical manner.

\section{Results}

Demographic data

A total of 70 consecutive patients were screened for inclusion, from May 2004 to May 2007. Finally, 36 pa- tients (32 male and 4 female patients) were included. All suffered from head-and-neck carcinoma (most of them squamous cells carcinomas), and their oncology therapy included radiation in doses ranging from 50-80 Gy, delivered in 5 periods. From this population at baseline (18 in the placebo group-mean age 54.3 $\pm 16.1,17$ males, 6 smokers- and 18 in the test group-mean age $49.4 \pm 15.4,15$ males, 3 smokers), 31 patients (16 in the placebo and 15 in the test group) completed the 2-week visit and 26 the 4-week visit (12 in the placebo and 14 in the test group). Reasons for drop-outs were: one patient in the placebo group died, one patient in the test group needed surgery and another had to be admitted at the Hospital. The rest of the drop-outs were related to the difficulties for patients to comply with the appointments due to their health-related problems.

Clinical outcome variables (Table 1)

Out of the two patients with mucositis at baseline in the placebo group, one showed degree 1 and the other degree 4 . The 5 positive patients in the test group showed degrees 1, 2, 3 (2 patients) and 4, respectively. After 2 weeks, 9 placebo patients experienced an increase in the degree of mucositis, and no changed was observed in 7 patients, with a median change of 1.5 score points.

Table 1. Changes in clinical outcome variables: degree of mucositis, plaque index and gingival index.

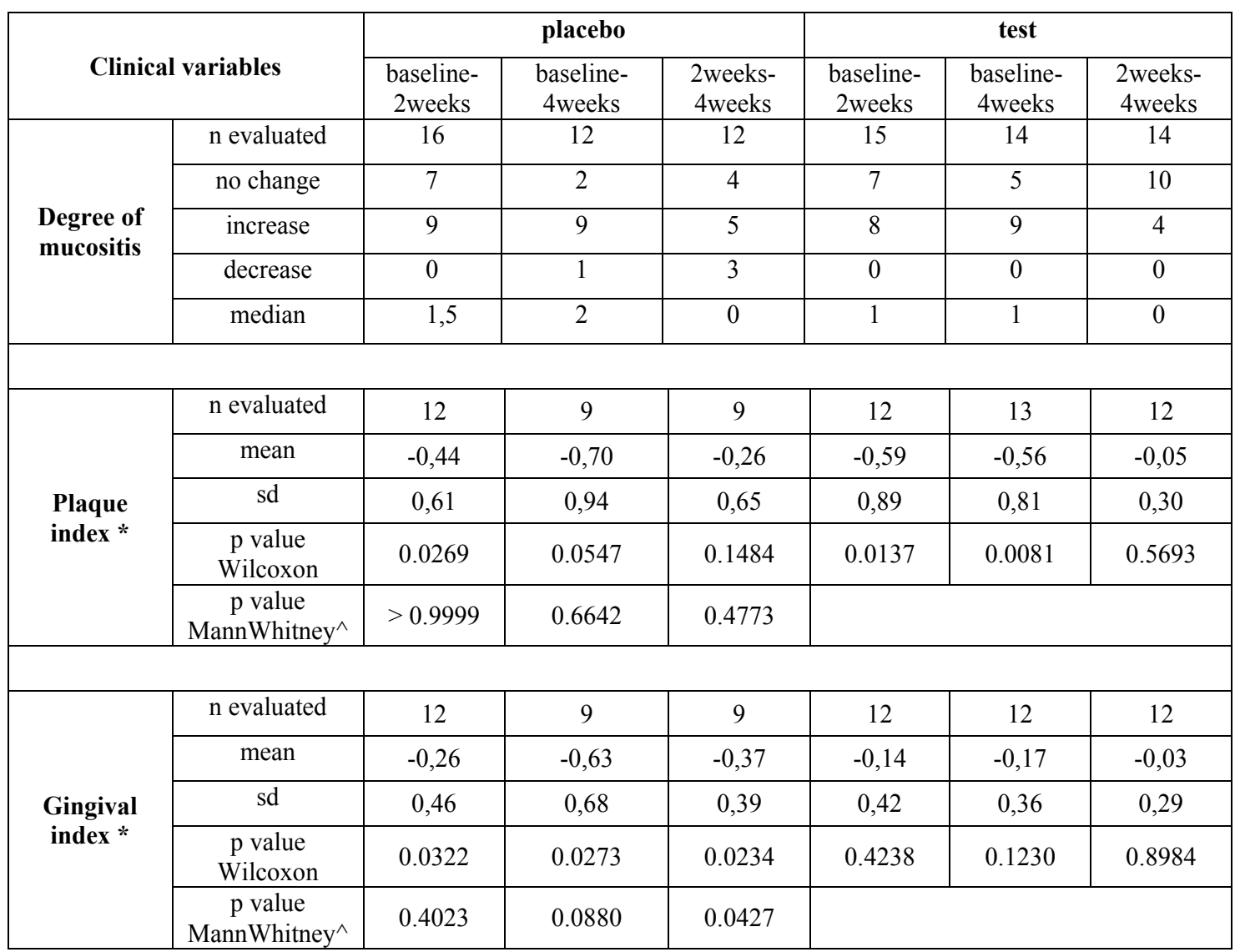

* Negative values mean decrease. ${ }^{\wedge}$ Inter group comparison. sd, standard deviation.In Bold, statistically significant differences. 
In the test group, 8 patients suffered an increase and 7 no change, being the median 1 . The statistical comparison showed no significant differences between the medians of the groups ( $p=0.381$ ), although higher mean values were observed in the placebo group (1.81 versus 1.20). After 4 weeks, 9 placebo patients demonstrated an increment from baseline, two remained unchanged and one showed a decrease. The median of the increase was 2 score points. In the test group, 9 showed increase and 5 no change, being the median change of 1 . No significant differences were detected $(\mathrm{p}=0.772)$. The mean change was similar (1.75 versus 1.71$)$. In regards to the changes between 2 and 4 weeks, in the placebo group, 5 patients increased, 3 decreased and 4 did not change. In the test group, no changes were detected for 10 patients and 4 experienced an increase. The median change was 0 , while the mean was higher for test patients $(0.64)$ than for placebo patients $(0.25)$. No significant differences were detected $(\mathrm{p}=0.779)$.

Plaque levels were significantly reduced in both groups after 2 weeks $(\mathrm{p}<0.05)$ and after 4 weeks $(\mathrm{p} \leq 0.05)$. When test and placebo groups were compared, differences were not statistically significant either at each study visit or in the changes between visits.

Gingivitis levels were reduced in both groups, but only reached the level of statistical significance in the control group $(\mathrm{p}<0.05)$. Differences between groups were statistical significant in the additional reduction between 2 and 4 weeks, corresponding to higher reduction in the control group.

\section{Salivary outcome variables (Table 2)}

Stimulated salivary flow was reduced in both groups from baseline to 2 weeks, although not reaching the level of significance. From 2 to 4 weeks, patients in the

Table 2. Changes in salivary outcome variables: referred hiposialosis, stimulated saliva and salivary $\mathrm{pH}$.

\begin{tabular}{|c|c|c|c|c|c|c|c|}
\hline \multirow{2}{*}{\multicolumn{2}{|c|}{ Salivary outcome variables }} & \multicolumn{3}{|c|}{ placebo } & \multicolumn{3}{|c|}{ test } \\
\hline & & \multirow{2}{*}{$\begin{array}{c}\text { baseline- } \\
\text { 2weeks } \\
15\end{array}$} & \multirow{2}{*}{$\begin{array}{c}\begin{array}{c}\text { baseline- } \\
\text { 4weeks }\end{array} \\
10\end{array}$} & \multirow{2}{*}{$\begin{array}{c}\begin{array}{c}\text { 2weeks- } \\
\text { 4weeks }\end{array} \\
10\end{array}$} & \multirow{2}{*}{$\begin{array}{c}\begin{array}{c}\text { baseline- } \\
\text { 2weeks }\end{array} \\
14\end{array}$} & \multirow{2}{*}{$\begin{array}{c}\begin{array}{c}\text { baseline- } \\
\text { 4weeks }\end{array} \\
14\end{array}$} & \multirow{2}{*}{$\begin{array}{c}\begin{array}{c}2 \text { weeks- } \\
\text { 4weeks }\end{array} \\
14\end{array}$} \\
\hline \multirow{6}{*}{$\begin{array}{l}\text { Changes in } \\
\text { referred } \\
\text { hiposialosis }\end{array}$} & evaluated & & & & & & \\
\hline & no change & 9 & 7 & 10 & 9 & 8 & 13 \\
\hline & increase & 6 & 3 & 0 & 5 & 6 & 1 \\
\hline & decrease & 0 & 0 & 0 & 0 & 0 & 0 \\
\hline & median & 0 & 0 & 0 & 0 & 0 & 0 \\
\hline & $\begin{array}{c}\mathrm{p} \text { value } \\
\text { chi-square }^{\wedge}\end{array}$ & 1 & 0,8307 & 1 & & & \\
\hline \multirow{7}{*}{$\begin{array}{c}\text { Changes in } \\
\text { stimulated } \\
\text { saliva in } \mathbf{m L} \\
*\end{array}$} & evaluated & 9 & 9 & 8 & 8 & 7 & 9 \\
\hline & mean & $-2,20$ & $-1,93$ & 0,11 & $-2,19$ & $-5,00$ & $-1,89$ \\
\hline & $\max$ & 0,1 & 0,5 & 1 & 4 & $-0,5$ & 0 \\
\hline & $\min$ & -9 & -8 & $-1,5$ & -11 & -14 & -5 \\
\hline & $\mathrm{sd}$ & 3,17 & 3,29 & 0,90 & 4,33 & 4,79 & 1,88 \\
\hline & $\begin{array}{c}\mathrm{p} \text {-value } \\
\mathrm{t} \text {-test }\end{array}$ & 0,07 & 0,11 & 0,73 & 0,26 & 0,03 & 0,02 \\
\hline & $\begin{array}{c}\text { p value } \\
\text { t-test }^{\wedge}\end{array}$ & 0,99 & 0,18 & 0,02 & & & \\
\hline \multirow{7}{*}{$\begin{array}{l}\text { Changes in } \\
\text { salivary pH }\end{array}$} & evaluated & 9 & 8 & 8 & 9 & 7 & 8 \\
\hline & $\mathrm{n}(-2)$ & 1 & 3 & 1 & 2 & 2 & 0 \\
\hline & $\mathrm{n}(-1)$ & 4 & 3 & 2 & 2 & 2 & 0 \\
\hline & $\mathrm{n}(0)$ & 4 & 2 & 4 & 5 & 3 & 8 \\
\hline & $\mathrm{n}(+1)$ & 0 & 0 & 1 & 0 & 0 & 0 \\
\hline & $\begin{array}{c}\mathrm{p} \text { value } \\
\text { chi-square }\end{array}$ & 0,22 & 0,69 & 0,44 & 0,46 & 0,71 & 0,003 \\
\hline & $\begin{array}{c}\text { p value } \\
\text { chi-square }^{\wedge}\end{array}$ & 0,57 & 0,76 & 0,15 & & & \\
\hline
\end{tabular}

* Negative values mean decrease. ${ }^{\wedge}$ Inter group comparison. sd, standard deviation. In Bold, statistically significant differences. 
test group showed an additional significant $(p=0.02)$ decrease. Differences between baseline and 4 weeks were also significant in this group $(\mathrm{p}=0.03)$. When both groups were compared, the differences in the changes between $2-4$ weeks were statistically significant $(\mathrm{p}=0.02)$.

Four patients in the placebo group and 7 patients in the test group referred hyposialosis at baseline. No significant differences were detected at baseline, 2 weeks or 4 weeks. Approximately one third of the patients in both groups ( 6 in control group, and 5 in the test group) reported an increase in hyposialia between baseline and 2 weeks, after the irradiation treatment started. No additional changes were detected from 2 to 4 weeks, except for one patient in the test group. No significant differences were detected between groups at each visit or in the changes between visits, although a higher level of hyposialosis was observed in test patients at baseline. With regards to salivary $\mathrm{pH}$, the buffer capacity of saliva decreased from baseline to 2 weeks in both groups. An additional decrease was observed for the control group from 2 to 4 weeks, while no changes were found in the test group. Differences between groups in the changes 2 weeks-4 weeks, were statistically significant $(p=0.003)$. However, it should be noted that the situation at baseline was also different, although not statistically significant, with a worse condition in the test group.

Adverse effects

No relevant adverse effects were reported in any group.

\section{Discussion}

The results of the present study have shown some benefits in using a non-alcohol, CHX and CPC mouth rinse in patients undergoing radiation therapy as part of the treatment of a head-and-neck cancer. These results, however, should be interpreted with caution, due to the heterogeneity of the patient sample and the inherent difficulties when enrolling patients with severe health conditions in clinical trials.

There are many confounding factors that may have influenced the results, such as:

- The total radiation dose and treatment regimen. In most patients it ranged between 50-70 Gy, fractioned in 5 weekly sessions of $2 \mathrm{~Gy} / \mathrm{session}$, aiming at reducing the secondary effects and at facilitating the oxygenation of the tumoral cells. Since radiation damage is anatomically site-specific, its toxicity is localized to the irradiated tissue volumes. The degree of damage will be dependent on factors related to the treatment regimen, such as the type of radiation used, the total administered dose, and the field size in relation to the fractionation. Even for HNT, the radiation will depend on the location and extension of the tumour, with important differences for a T1 N0 M0 as compared with a T3 N2 M0 of tongue, or between a cancer of cavum and a tongue base carcinoma.
- The tumour type, clinical stage, histology, location, extension... and the existence of previous or concomitant chemotherapy also provides a high degree of heterogeneity in the patient sample.

- The patient systemic status usually deteriorated during the oncology therapy. The ensuing secondary effects, such as mucositis were associated with pain, haemorrhage and infection, which precluded a normal diet and an adequate oral hygiene. Even the incidence of candidiasis in a neutropenic patient may lead to hospitalization and to the temporary suspension of the radiotherapy. These problems precluded some patients from continuing the study and therefore, to comply with the programmed study visits.

- Concomitant oral care, since patients were using, as part of the hospital protocols, other mouth rinses $(0.1 \%$ hexetidine with and without alcohol,...) with different active ingredients that may not be considered as very active, but could have influenced the results.

- Patient habits, such as oral hygiene habits, alcohol consumption, smoking habits...

CHX mouth rinses in the prevention of oral mucositis have been evaluated in at least 9 randomized clinical trials $(2,11-14,17-20)$, but the outcomes were quite different. From these studies, 7 were included in a metaanalysis (2), that showed no effect of chlorhexidine in the prevention of mucositis in chemotherapy and radiotherapy patients (odds ratio $0.7 ; 95 \%$ confidence interval $0.43-1.12$ ).

Out of these 7 studies, three evaluated the effect of $\mathrm{CHX}$ in patients under radiotherapy. In one of them (12), 30 patients receiving high-dose head-and-neck radiation therapy were evaluated. A $0.12 \%$ CHX digluconate mouth rinse was evaluated $(15 \mathrm{~mL}, 3$ times a day) and no differences were observed in oral mucositis ("presence of mucositis" in categories ranging 0-4) between the control and $\mathrm{CHX}$ groups, although reductions in oral microflora were observed in the CHX group. Another study (13) suggested that a CHX mouthwash was detrimental in the management of mucositis. Finally, in the third study $(15,19)$, a $0.1 \%$ CHX mouthrinse was evaluated, and 30 HNT patients were included, who rinsed four times per day with the assigned product. No differences were observed between the two study groups in the development and severity of mucositis.

The results of this clinical investigation, however, suggest clinical benefits in the use of the provided antiseptic oral rinse. One of the explanations for these positive results may be related to the improved formulation of the tested product that has shown higher activity (16). The potential benefit of the prophylactic rinse with the tested product, rather than on a direct effect upon oral mucositis, may be through the control of oral microorganisms together with the reduction in the occurrence of oropharyngeal candidosis. In addition, it is important 
to provide these patients with supplementary supportive measures including dietary recommendations, such as strong emphasis on maintaining the nutrition and the use of a soft diet and the avoidance of irritants such tobacco, alcohol or spices. It should be pointed out that the results of the present study should be interpreted with caution, due to all the limiting factors previously enumerated and the reduced sample size.

\section{Conclusions}

We can conclude that, within the limitations of the small sample size, this study suggests that the use of a $0.12 \%$ $\mathrm{CHX}$ and $0.05 \% \mathrm{CPC}$ mouth rinse may lead to some improvements in clinical parameters in patients irradiated for head-and-neck cancer.

\section{References}

1. Caribé-Gomes F, Chimenos-Küstner E, López-López J, FinestresZubeldia F, Guix-Melcior B. Dental management of the complications of radio and chemotherapy in oral cancer. Med Oral. 2003;8:178-87. 2. Stokman MA, Spijkervet FK, Boezen HM, Schouten JP, Roodenburg JL, de Vries EG. Preventive intervention possibilities in radiotherapy- and chemotherapy-induced oral mucositis: results of metaanalyses. J Dent Res. 2006;85:690-700.

3. Puyalt Casado M, Jiménez Martinez C, Chimenos Küstner E, López López J, Juliá A. A protocol for the evaluation and treatment of oral mucositis in patients with hematological malignancies. Med Oral. 2003;8:10-8.

4. Scully C, Sonis S, Diz PD. Oral mucositis. Oral Dis. 2006;12:229 41.

5. Stokman MA, Spijkervet FK, Burlage FR, Dijkstra PU, Manson WL, de Vries EG, et al. Oral mucositis and selective elimination of oral flora in head and neck cancer patients receiving radiotherapy: a double-blind randomised clinical trial. Br J Cancer. 2003;88:1012-6. 6. Worthington HV, Clarkson JE, Eden OB. Interventions for preventing oral mucositis for patients with cancer receiving treatment. Cochrane Database Syst Rev. 2007;4:CD000978.

7. Clarkson JE, Worthington HV, Eden OB. Interventions for treating oral mucositis for patients with cancer receiving treatment. Cochrane Database Syst Rev. 2007;2:CD001973.

8. Keefe DM, Schubert MM, Elting LS, Sonis ST, Epstein JB, RaberDurlacher JE, et al. Updated clinical practice guidelines for the prevention and treatment of mucositis. Cancer. 2007;109:820-31.

9. Addy M, Moran JM. Clinical indications for the use of chemical adjuncts to plaque control: chlorhexidine formulations. Periodontol 2000. 1997; 15:52-4.

10. Wijers OB, Levendag PC, Harms ER, Gan-Teng AM, Schmitz PI, Hendriks WD, et al. Mucositis reduction by selective elimination of oral flora in irradiated cancers of the head and neck: a placebocontrolled double-blind randomized study. Int J Radiat Oncol Biol Phys. 2001;50:343-52.

11. Dodd MJ, Larson PJ, Dibble SL, Miaskowski C, Greenspan D, MacPhail L, et al. Randomized clinical trial of chlorhexidine versus placebo for prevention of oral mucositis in patients receiving chemotherapy. Oncol Nurs Forum. 1996;23:921-7.

12. Ferretti GA, Raybould TP, Brown AT, Macdonald JS, Greenwood M, Maruyama Y, et al. Chlorhexidine prophylaxis for chemotherapyand radiotherapy-induced stomatitis: a randomized double-blind trial. Oral Surg Oral Med Oral Pathol. 1990;69:331-8.

13. Foote RL, Loprinzi CL, Frank AR, O'Fallon JR, Gulavita S, Tewfik HH, et al. Randomized trial of a chlorhexidine mouthwash for alleviation of radiation-induced mucositis. J Clin Oncol. 1994;12:2630-3.
14. Pitten FA, Kiefer T, Buth C, Doelken G, Kramer A. Do cancer patients with chemotherapy-induced leukopenia benefit from an antiseptic chlorhexidine-based oral rinse? A double-blind, block-randomized, controlled study. J Hosp Infect. 2003;53:283-91.

15. Spijkervet FK, Van Saene HK, Panders AK, Vermey A, Van Saene JJ, Mehta DM, et al. Effect of chlorhexidine rinsing on the oropharyngeal ecology in patients with head and neck cancer who have irradiation mucositis. Oral Surg Oral Med Oral Pathol. 1989;67:154-61. 16. Herrera D, Roldán S, Santacruz I, Santos S, Masdevall M, Sanz M. Differences in antimicrobial activity of four commercial $0.12 \%$ chlorhexidine mouthrinse formulations: an in vitro contact test and salivary bacterial counts study. J Clin Periodontol. 2003;30:307-14.

17. Cheng KK, Chang AM. Palliation of oral mucositis symptoms in pediatric patients treated with cancer chemotherapy. Cancer Nurs. 2003;26:476-84.

18. Dodd MJ, Dibble SL, Miaskowski C, MacPhail L, Greenspan $\mathrm{D}$, Paul SM, et al. Randomized clinical trial of the effectiveness of 3 commonly used mouthwashes to treat chemotherapy-induced mucositis. Oral Surg Oral Med Oral Pathol Oral Radiol Endod. 2000;90:39-47.

19. Spijkervet FK, Van Saene HK, Van Saene JJ, Panders AK, Vermey A, Mehta DM. Mucositis prevention by selective elimination of oral flora in irradiated head and neck cancer patients. J Oral Pathol Med. 1990;19:486-9.

20. Epstein JB, Vickars L, Spinelli J, Reece D. Efficacy of chlorhexidine and nystatin rinses in prevention of oral complications in leukemia and bone marrow transplantation. Oral Surg Oral Med Oral Pathol. 1992;73:682-9.

\section{Acknowledgements}

The authors want to thanks the personnel working at the Oncological Radiotherapy Service "Hospital 12 de Octubre" (Madrid, Spain). 